\title{
Investigation of water droplets, kerosene and ethanol deformation in the air
}

\author{
Roman Volkov, Alena Zhdanova, and Pavel Strizhak ${ }^{\mathrm{a}}$ \\ National Research Tomsk Polytechnic University, 30, Lenin Avenue, 634050 Tomsk, Russia
}

\begin{abstract}
Experimental investigation of liquid droplets deformation features during motion through the air with velocities up to $5 \mathrm{~m} / \mathrm{s}$ was executed. Typical liquids (water, kerosene, ethanol) were observed. More than ten successive "strain cycles" were investigated during the droplets motions of the $1 \mathrm{~m}$ distance thought the air. Characteristics times of droplet changes from form to form, and possible "deformation modes" were determined.
\end{abstract}

\section{Introduction}

Results of numerical researches [1, 2] have shown that the factor of liquid droplets form influence on integrated characteristics of their movement through the high-temperature gas area is defining. Continuous variation of movement resistance coefficient $\left(\mathrm{c}_{\chi}\right)$ in time should be considered to draw near the model processes to real ones [1,2]. The nature of this variation and its dependence on external and internal factors has not been defined fully.

It has been identified in experimental and theoretical works [3-6], that the motion of droplets in gas areas and liquids is the specific sequence of "strain cycles". The "strain cycle" means the time interval $t_{\mathrm{d}}$, when the droplet consistently twice takes the form close to the size, the position of the symmetry axis and other features during its motion. Six characteristic configurations of water droplets with glycerin admixtures at their free falling thought the air for the first "strain cycle" were marked in [4].

Videogams of experiments [7-9] allows carrying the inference that the velocities of droplets movement may significantly influence on the droplets form and the nature of their deformation. High-velocity video registration of droplets movement in gas areas (with various properties, sizes and velocities) is of interest. It will allow establishing the most typical forms of droplets to use at corresponding processes simulation and transition times from one form to another during movement. It is understandable that, it is necessary to investigate the regularities of deformation not only within the first, but also the group of the subsequent "strain cycles" to extend the possible supplements (for example, perspective gas- and vapor-droplet technologies $[9,10])$ of received results in contrast to the experiments [3-6].

The purpose of the work is experimental research of features of water droplets, kerosene and ethanol deformation in the air.

\footnotetext{
${ }^{a}$ Corresponding author: pavelspa@tpu.ru
}

This is an Open Access article distributed under the terms of the Creative Commons Attribution License 4.0, which permits unrestricted use, distribution, and reproduction in any medium, provided the original work is properly cited. 


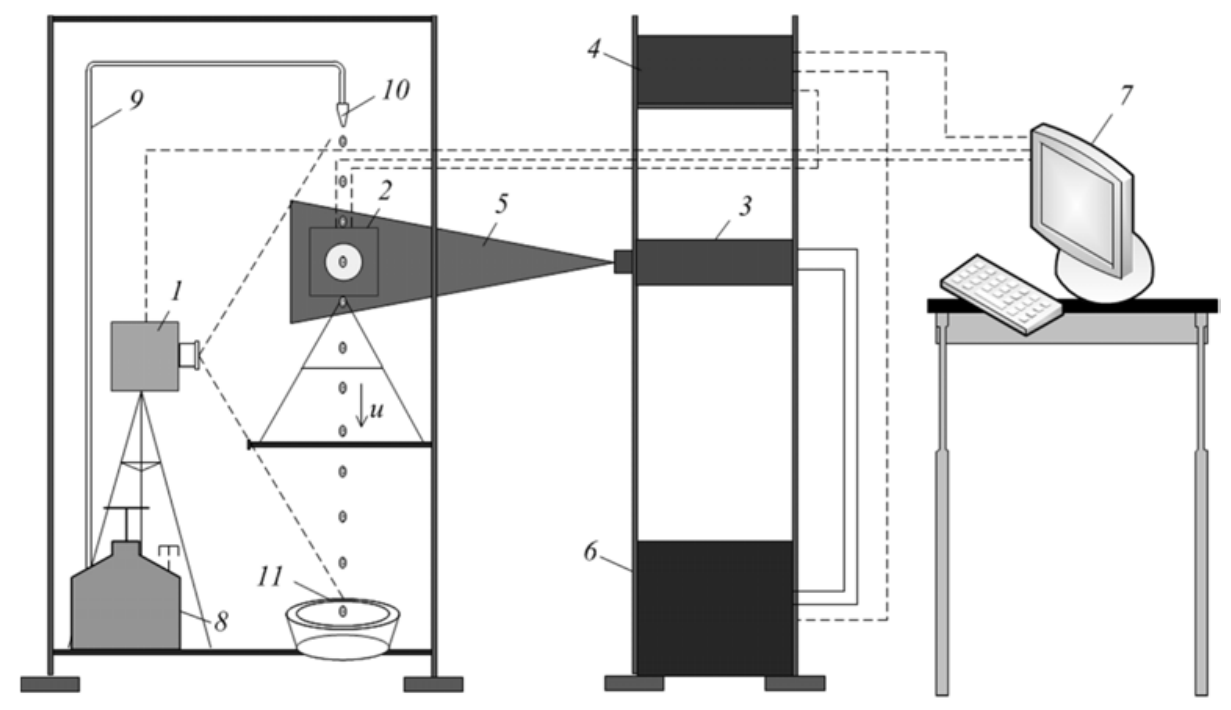

Figure 1. A scheme of experimental setup: 1 - high-velocity digital camera, 2 - cross-correlation digital camera, 3 -double pulsed solid-state laser, 4 - synchronizer of personal computer (PC), cross-correlation digital camera and laser, 5 - light "pulse", 6 - laser generator, 7 - PC, 8 - vessel with experimental liquid, 9 - channel of experimental liquid supply, 10 - dosing device, 11 - catcher.

\section{Experimental setup and methods}

Scheme of experimental setup is shown in Fig. 1. The setup (on the basic measuring equipment) is similar to that used in $[11,12]$ with using of "tracer" visualization optical "Particle Image Velocimetry" [13] and "Interferometric Particle Imaging" [14] methods. In particular it is used (Fig. 1): digital video camera 1 with figure format of $1024 \times 1024$ pixels, frame frequency up to $10^{5}$ per second; crosscorrelation digital camera 2 with figure format of $2048 \times 2048$ pixels, minimal delay between two sequence frames not more than $5 \mu \mathrm{s}$; double pulsed solid-state laser 3 with wave-length $532 \mathrm{~nm}$, energy in impulse not less than $70 \mathrm{~mJ}$, impulse time not more than $12 \mathrm{~ns}$, recurrence frequency not more than $15 \mathrm{~Hz}$; synchronizing processor 4 with signal sampling not more than $10 \mathrm{~ns}$.

Initial characteristic droplet sizes of investigated liquids (water, ethanol, kerosene) were changed in the range from 3 to $6 \mathrm{~mm}$ in experiments. Its registered diameter $\left(d_{0}\right)$ at an outlet from the dosing device 10 was accepted as such size.

Initial velocity of droplet movement (ejection by the dosing device 10 ) $u_{0}=0 \div 3 \mathrm{~m} / \mathrm{s}$ was one of the basic varying parameters in experiments. Droplets velocities $u$ were attained $5 \mathrm{~m} / \mathrm{s}$ in video registration areas from the dosing device 10 to catcher 11 (Fig. 1).

Maximum Weber's numbers were composed $\mathrm{We}_{\operatorname{maxw}}=u_{\max }^{2} d_{\max } \rho_{\mathrm{a}} / \sigma_{\mathrm{lw}}=52 \cdot 6 \cdot 10-3 \cdot 1.5 /$ $0.0618 \approx 3.64, \mathrm{We}_{\operatorname{maxs}}=u_{\max }^{2} d_{\max } \rho_{\mathrm{a}} / \sigma_{l s} \approx 9.86, \mathrm{We}_{\operatorname{maxk}}=u_{\max }^{2} d_{\max } \rho_{a} / \sigma_{\mathrm{lk}} \approx 7.78$ in experiments under maximum air density $\rho_{\mathrm{a}} \approx 1.5 \mathrm{~kg} / \mathrm{m}^{3}$ and surface tension coefficients of water, ethanol and kerosene $\sigma_{\mathrm{lw}}=0.0618 \mathrm{~kg} / \mathrm{s}^{2}, \sigma_{\mathrm{ls}}=0.0228 \mathrm{~kg} / \mathrm{s}^{2} \sigma_{\mathrm{lk}}=0.0289 \mathrm{~kg} / \mathrm{s}^{2}$ in considered conditions. It has been identified in experiments [8-10] that the probability of liquid droplet breakup is high at Weber's numbers more than 10 . Generally, value of $\mathrm{We}_{\text {lim }}=10$ is considered as a maximum permissible one when the solidity preservation of liquids droplets during motion in gas area is possible.

Breakup conditions of single water, ethanol and kerosene droplets at $d_{0}=3 \div 6 \mathrm{~mm}, u_{0}=0 \div$ $3 \mathrm{~m} / \mathrm{s}$ and their $1 \mathrm{~m}$ distance motion through the air under the action of gravitational forces weren't registered in the conducted experiments. Therefore it is reputed, that the experiment conditions are corresponded to moderate (We $<\mathrm{We}_{\mathrm{lim}}$ ) Weber's numbers. 


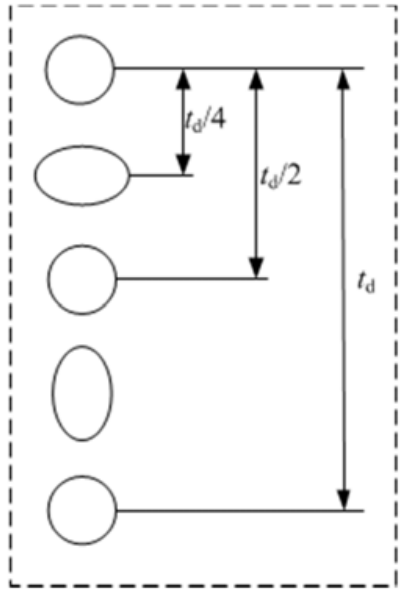

(a)

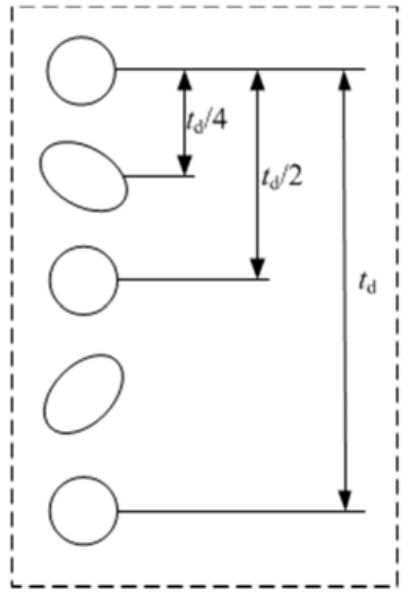

(b)

Figure 2. The conventional images of liquid droplets at realization of the first (a) and second (b) "deformation modes".

Droplets of one of the investigated liquids with defined initial sizes $d_{0}$ and velocities $u_{0}$ were emanated from the dosing device 10 and flew the distance of $1 \mathrm{~m}$ in air through the video registration field to the catcher 11 (Fig. 1). The process of droplets movement was registered by the digital camera 1 (with frame frequency up to $10^{4}$ per second). Ten experiments were conducted for the fixture sizes and velocities of droplets under other fixed conditions. Fields corresponding to "strain cycles" were marked on the personal computer (PC) 7 after processing of videograms. Droplets were assumed the form similar (by configuration, symmetry axis position and characteristics dimensions) to initial one during these "strain cycles". The distance between the dosing device 10 and the catcher 11 was detached on group of fields characterizing the relevant "strain cycles". Measurement of the droplet sizes was conducted with use of the cross-correlation digital camera 2, solid-state laser 3 and synchronizer 4 for marked "strain cycles". Ten experiments with identical sizes and velocities of droplets were accomplished. Images of droplet were fixed only for one or several successive "strain cycles". Each videogram was divided on the estimated domains with dimensions $32 \times 32$ pixels. The scaling coefficient $\gamma$ was calculated in consistent with recommendations $[13,14]$. The values of $\gamma$ were changed in $0.01 \div 0.1 \mathrm{~mm} /$ pixel interval. The maximum diameter of droplets in pixels were determined and then the conversion in millimeters with the application of coefficient $\gamma$ was completed with using the algorithms [13, 14] and techniques [12].

\section{Results and discussion}

Two "deformation modes" differing by sequential forms of droplets (Fig. 2) have been established when videograms processing of the executed experiments. The droplet from the initial state near to spherical one is "flattened" and assumes the "plate" form and then it is stretched along-track direction (then these forms are repeated cyclically) at realization of the first mode (Fig. 2a). The second mode (Fig. 2b) exactly may be called as a "vortical" one. Rotation of droplet in the ellipsoid form about its own center of inertia during its motions is well visible at realization of the second mode.

The slightly different conditions of liquid droplets ejection by the dosing device 10 (Fig. 1) in the conducted experiments are the main cause of revealed "deformation modes" realization. The first mode (Fig. 2a) is implemented at the "optimum" transverse ejection of droplets. The second mode (Fig. 2b) is 


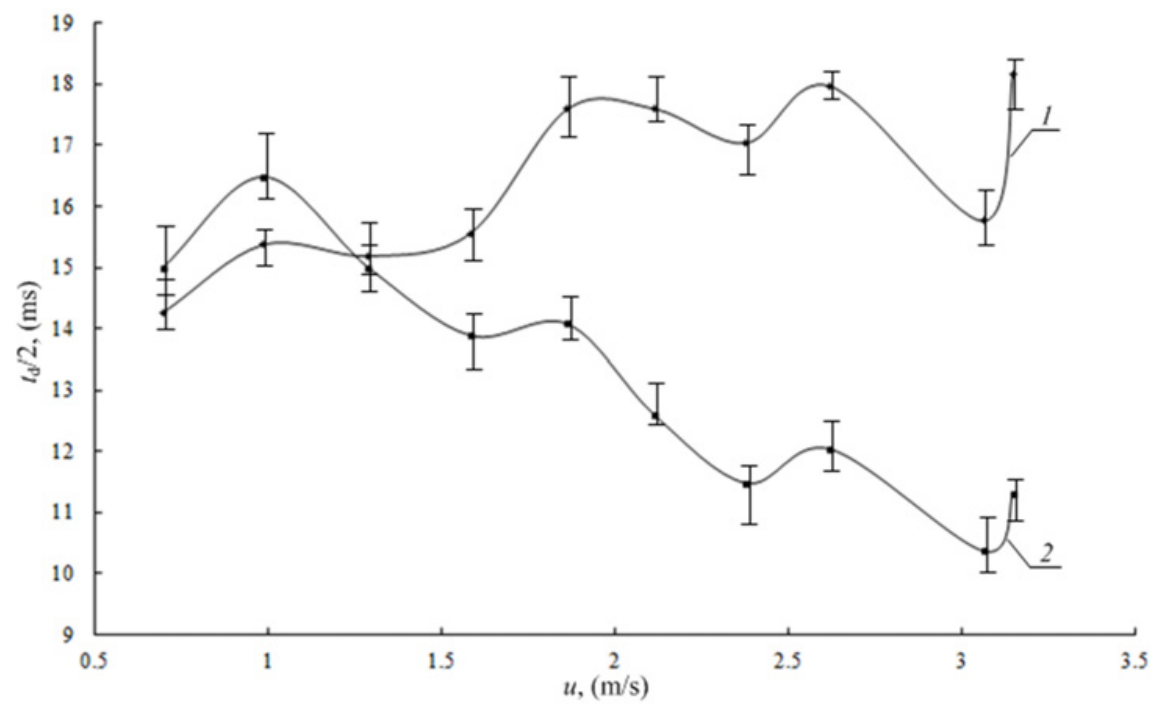

(a)

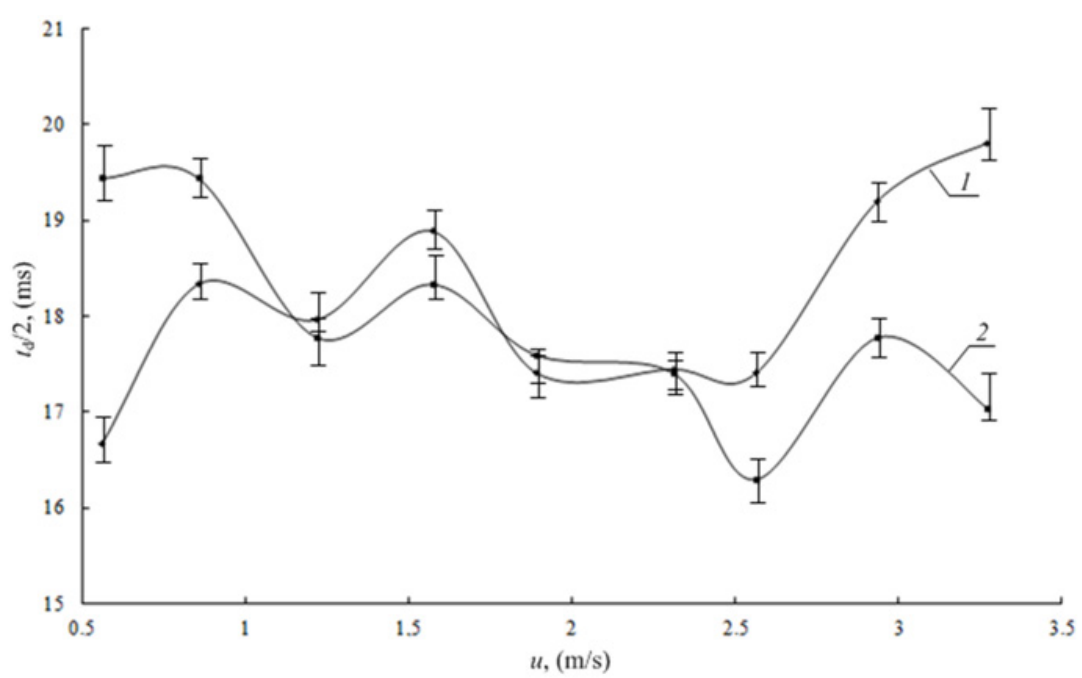

(b)

Figure 3. A change in "semicircles" times during the realization process of the first $\left(\mathrm{a}-d_{0}=4 \mathrm{~mm} ; \mathrm{b}-d_{0}=\right.$ $4.5 \mathrm{~mm}$ ) "deformation mode" of ethanol droplets at their various initial droplet sizes and velocities at the "strain cycles" inlet: 1 - the first "semicircle", 2 - the second "semicircle".

implemented if the presence of even small canting angle deviation (more then $2^{0}$ ) of the emissive dosing device channel relatively $90^{\circ}$ to the basis of the catcher 11 .

The features have been established while processing of characteristic droplet "strain cycles" for investigated liquids at implementation process of the first and second modes. These features related to pretty significant time differs both of "semicircles" and "quarter cycles" (the modification from the "plate" form to sphere, from sphere to ellipsoid form stretched along-track droplet motion and in reverse). Times of "semicircles" during the realization process of the first (Fig. 2a) ethanol droplets "deformation mode" with their various initial sizes and velocities at the inlet of "strain cycles" are presented in Fig. 3. 


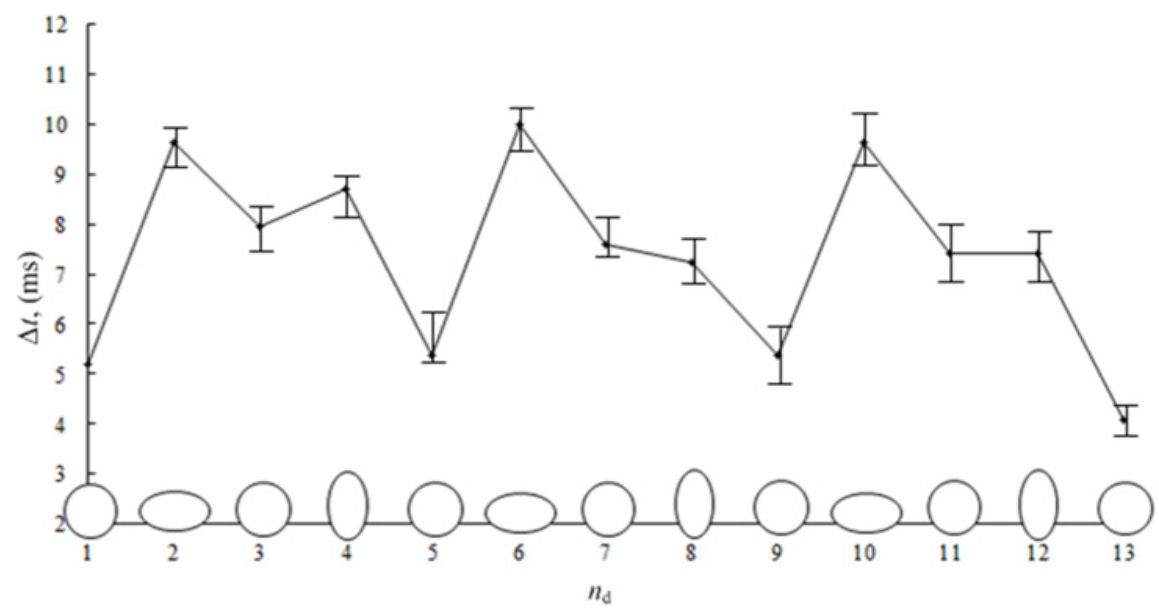

Figure 4. Transition times of the ethanol droplets from form to form within limits of fifth, sixth and seventh strains at realization of the first "deformation mode".

Times of "semicircles" are differed sufficiently significantly at realization of the first "deformation mode". Immensely nonsignificant and "fluctuated" difference between characteristic times "semicircles" can be noted for the second mode.

It is expedient to observe the transition processes from one droplet form to another at established modes realization to explain the revealed features.

Figure 4 shows the experimental values of such transitions times for several consequents "strain cycles" of ethanol droplets at realization of the first mode.

The parameter $n_{\mathrm{d}}$ characterizes serial number of droplet forms within the frame of the observed cycles (numbers of droplet forms 1-5 correspond to the fifth, 5-9 - to the sixth, 9-13 - to the seventh of "strain cycles", respectively).

It is sufficiently clearly seen (Fig. 4) that the transition times from the sphere form to "plate" one, from the "plate" to sphere and from the sphere to ellipsoid stretched along-track direction of droplets are differed. These contrasts are reputed to be significant factors when the times are $t_{\mathrm{d}} \ll 1 \mathrm{~s}$.

It has been established that transition times from spherical droplets to ellipsoids and "plates" are more than times characterizing the reverse transfers by $15 \div 20 \%$. Revealed phenomenon is due to corresponding activity of mass and inertial forces. The resistance forces affected on the droplets of spherical form during their motion in gas areas are less than ones at "ellipse-like" configurations of droplet surface. As a consequence the "slowdown" of ellipsoid form droplets and all the more the "plate" ones by the gas area is more intensive then "slowdown" of spherical droplets. This involves to the depression of the droplets drive-up phenomenon under the gravitational force influence and the increase of "plate - ellipsoid" transition times. The resistance force decreases slightly and the droplet is accelerated - of transition time decreases (Fig. 4) under reverse transfer. The established regularity traces back very plain with increase of passed "strain cycles" number despite the transition time intervals from one droplet form to another are decreased significantly as the values of $t_{\mathrm{d}}$ in all.

\section{Conclusions}

The experiments for analysis of possible liquid droplets "strain cycles" in gas area (at Weber's moderate numbers) have been executed. The conducted experiments allowed establishing the real droplet forms, their number, times between forms and other characteristics of typical "strain cycles". The controlling 
influence on the main parameter of typical "strain cycles" in the air for widely used liquids (water, ethanol, kerosene) has been established. Not only the liquid properties (surface-tension, viscosity, density) but sizes, and also droplet motion velocity have this influence.

Videogams of conducted experiments show that the droplet spherical form is founded not oftener than "ellipsoid" or "plate" ones during their motion in air. Droplets have the spherical form during not more than $15 \%$ of the total time at moving in video registration area.

This work was financially supported by the Ministry of Education and Science of the Russian Federation (the state contract 2.1321.2014) and subsidy funds as part of the implementation of Competitive growth program of the National Research Tomsk Polytechnic University.

\section{References}

[1] O.V. Vysokomornaya, G.V. Kuznetsov, P.A. Strizhak, Journal of Engineering Physics and Thermophysics 86, 1 (2013)

[2] P.A. Strizhak, Journal of Engineering Physics and Thermophysics 86, 4 (2013)

[3] A. Wierzba, Experiments in Fluids 9, 1 (1990)

[4] V. V. Dubrovskii, A. M. Podvysotskii, A. A. Shraiber, Journal of Engineering Physics 58, 5 (1990)

[5] L.P. Hsiang, G.M. Faeth, International Journal of Multiphase Flow 19, 5 (1993)

[6] A. A. Shreiber, A. M. Podvisotski, V. V. Dubrovski, Atomization and Sprays 6, 6 (1996)

[7] S.S. Hwang, Z. Liu, R.D. Reitz, Atomization Sprays 6 (1996)

[8] D.R. Guildenbecher, P.E. Sojka, Atomization Sprays 21 (2011)

[9] A.K. Flock, D.R. Guildenbecher, J. Chen, P.E. Sojka, H.J. Bauer, International Journal of Multiphase Flow 47 (2012)

[10] J.E. Sprittles, Y.D. Shikhmurzaev, Physic of Fluids 24, 122105 (2012)

[11] A.V. Zakharevich, P.A. Strizhak, Journal of Engineering Thermophysics 22, 2 (2013)

[12] R.S. Volkov, O.V. Vysokomornaya, G.V. Kuznetsov, P.A. Strizhak, Journal of Engineering Physics and Thermophysics 86, 6 (2013)

[13] J. Westerweel, Measurement Science and Technology 8 (1997)

[14] N. Damaschke, H. Nobach, C. Tropea, Experiments in Fluids 32, 2 (2002) 